\title{
Parthenogenetic embryonic stem cells with H19 siRNA-mediated knockdown as a potential resource for cell therapy
}

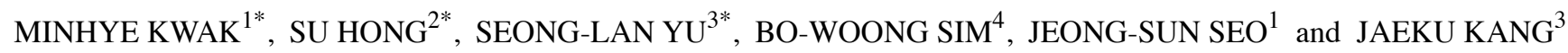 \\ ${ }^{1}$ Ilchun Genomic Medicine Institute, MRC, Department of Biochemistry and Molecular Biology, Seoul National University \\ College of Medicine, Seoul 110-799; ${ }^{2}$ Department of Bio and Brain Engineering, KAIST, Daejeon 305-701; ${ }^{3}$ Department \\ of Pharmacology, College of Medicine, Konyang University, Daejeon 302-718; ${ }^{4}$ National Primate Research \\ Center, Korea Research Institute of Bioscience and Biotechnology, Ochang 363-883, Republic of Korea
}

Received September 9, 2011; Accepted October 31, 2011

DOI: $10.3892 /$ ijmm.2011.838

\begin{abstract}
Embryonic stem (ES) cells are used in cell therapy and tissue engineering due to their ability to produce different cells types. However, studies of ES cells that are derived from fertilized embryos have raised concerns about the limitations imposed by ethical and political considerations. Therefore, many studies of stem cells use the stem cells that are derived from unfertilized oocytes and adult tissue. Although parthenogenetic embryonic stem (ESP) cells also avoid ethical and political dilemmas and can be used in cell-based therapy, the ESP cells exhibit growth retardation problems. Therefore, to investigate the potential for muscle growth from genetically modified ESP cells, we established four ES cell types, including normal embryonic stem (ESN) cells, ESP cells, ESP cells that overexpress the insulin-like growth factor 2 (Igf2) gene (ESI) and ESP cells with down-regulated H19 gene expression (ESH). Using these cells, we examined the expression profiles of genes that were related to imprinting and muscle using microarrays. The gene expression patterns of ESI and ESH cells were similar and were more closely related to the ESN pattern than that of the ESP cells. Differentiated ESH cells exhibited increased expression of bone morphologic protein 4 (BMP4), which is a mesoderm marker, compared with the differentiated ESI cells. We showed that Igf 2 expression was induced by $\mathrm{H} 19$ silencing in the ESP cells via hypermethylation of the H19 imprinting control region 1 (ICR1). Moreover, the proportion of ESH-derived
\end{abstract}

Correspondence to: Dr Jeong-Sun Seo, Department of Biochemistry and Molecular Biology, Seoul National University College of Medicine, Seoul 110-799, Republic of Korea

E-mail: jeongsun@snu.ac.kr

Dr Jaeku Kang, Department of Pharmacology, College of Medicine, Konyang University, Daejeon 302-718, Republic of Korea

E-mail: jaeku@konyang.ac.kr

*Contributed equally

Key words: parthenogenetic embryonic stem cells, insulin-like growth factor 2, H19, cell-based therapy, muscular atrophy chimera was slightly higher than those produced from the ESP cells. In addition, we detected increased cell proliferation in the MEF cells following H19 knock-down. These results indicate that the ESH cells may be a source of cell-based therapy for conditions such as muscular atrophy.

\section{Introduction}

Embryonic stem (ES) cells are utilized in a wide range of cell therapy and tissue engineering applications due to their pluripotency, being able to differentiate into all types of adult cells (1). However, the destruction of embryos involved in ES cell research has raised inevitable ethical, legal, religious and political concerns. The numbers of studies evaluating induced pluripotent stem (iPS) cells that are derived from adult somatic cells have recently increased to compensate for the restricted use of ES cells. However, there are serious safety concerns because iPS cells can enter tumorigenesis at a faster rate than the ES cells (2). Parthenogenetic embryonic stem (ESP) cells have been considered as a resource for cell therapy and may reduce immune rejection due to the homozygosity of the major histocompatibility complex (MHC) alleles $(3,4)$. The ESP cells that are derived from unfertilized oocytes have a similar differentiation profiles as the ES cells in many animals, including humans (5), and may avoid the ethical and political concerns encountered when using ES cells that are derived from fertilized oocytes.

Duchenne and Becker muscular dystrophies (DMD and BMD) are genetic disorders that affect males and are characterized by decreased expression of the dystrophin gene located at Xp21 in muscle, and subsequent muscle weakness and atrophy from the loss of muscle mass (6). Many researchers are developing cell-based therapies using ES, iPS and adult stem cells to induce skeletal muscle regeneration (7). Bhagavati and $\mathrm{Xu}(8)$ have demonstrated that transplanted ES cells in dystrophic mice (mdx) generated skeletal muscle. Moreover, Kamochi et al (9) have suggested that myocyte precursors that are derived from insulin-like growth factor 2 (Igf2)-tranfected ES cells were more effective in the repair of damaged muscle and myopathy compared with those derived from untransfected ES cells. Cell therapies using ES cells have problems concerning immune rejection responses. In diseases 
with X-linked recessive inheritance such as DMD, females are asymptomatic when only one $\mathrm{X}$-chromosome is defective. Therefore, pluripotent ESP cells may be used as a candidate for cell-based therapies to treat X-linked disorders. The use of ESP cells is limited by lack of the expression of paternally imprinted genes that are associated with fetal and placental growth due to the absence of the paternal genome. Therefore, parthenotes cannot develop to term $(10,11)$. Kono et al (12) have demonstrated that parthenogenetic mice with a $13-\mathrm{kb}$ deletion in the maternal imprinting gene $\mathrm{H} 19$, which is located on the same chromosome as the Igf2 gene, could develop to term. However, this molecular mechanism is poorly understood.

The Igf2 gene plays an important role in the development of mesoderm-derived bone, muscle and skin (13-15). In addition, the overexpression and expression of the maternal copy of Igf2 may contribute to ovarian cancer (16).

Therefore, we established and characterized genetically modified ESP cell lines, Igf2 over-expressing (ESI) and H19 down-regulated (ESH) ESP cells, to investigate their potential use in the cell-based therapies for muscular atrophy.

\section{Materials and methods}

Animals. C57BL/6J and DBA mice (Jackson Laboratory, USA) were purchased and cared for in accordance with the Guidelines for the Care and Use of Lab Animals. All procedures were performed aseptically and were approved by the Experimental Animal Committee of the Seoul National University in Korea.

Lentiviral vector construction. The Igf2 over-expressing lentiviral vector was constructed by inserting Igf 2 cDNA into the $X b a \mathrm{I}$ and EcoRI restriction sites of the shLenti2.4G lentiviral vector (Macrogen Inc., Korea). The shRNA-expressing lentiviral vector that was used for RNAi targeting of H19 mRNA was constructed by inserting the synthetic doublestrand oligonucleotides (5'-CGGAATTCGTCCGTCTCGTT CTGAATCAAttcaagagaTTGATTCAGAACGAGACGGAC TTTTTGATATCTAGACA-3') into the EcoRI and $X b a I$ restriction sites of the shLenti2.4G lentiviral vector (Macrogen Inc.). These constructed lentivial vectors were verified by nucleotide sequencing.

Derivation of parthenogenetic embryonic stem cells and cell culture. Oocytes were collected from B6D2F1 hybrid mice and treated for $6 \mathrm{~h}$ in $\mathrm{Ca}^{2+}$-free $\mathrm{CZB}$ media containing $10 \mathrm{mM} \mathrm{SrCl}_{2}$ (Sigma, USA) and $5 \mu \mathrm{g} / \mathrm{ml}$ cytochalasin B (Sigma) to induce oocyte activation. After activation, the oocytes were washed and cultured in $\mathrm{KSOM}$ media in an incubator with $5 \% \mathrm{CO}_{2}$ at $37^{\circ} \mathrm{C}$. All ES cell lines were established as previously described (17). To construct ESP cells that overexpress Igf2 (ESI) and that express a reduced level of H19 (ESH), the ESP cells were infected with a lentivirus containing the appropriate shLenti2.4G lentiviral vectors (Macrogen Inc.). To select transfectants, the colonies were treated using selection media containing puromycin (DMEM/10\%FBS/1,000 ng/ml puromycin) for 7 days.

In vitro differentiation of ES cells. To induce spontaneous differentiation of the ES cells in vitro, undifferentiated ES cells were trypsinized, and cultured in suspension on petri dishes containing differentiation media [DMEM supplemented with
$20 \%$ FBS, penicillin $(100 \mathrm{U} / \mathrm{ml}) /$ streptomycin $(100 \mu \mathrm{g} / \mathrm{ml})$, $1 \mathrm{X}$ nonessential amino acids and $100 \mu \mathrm{M} \beta$-mercaptoethanol without LIF]. The aggregated embryonic bodies (EB) were transferred to new petri dishes containing fresh differentiation media, which was replaced every day. The cells were harvested for RNA extraction after 10 days in culture.

RNA isolation. Total-RNA was extracted with TRIzol reagent (Invitrogen, USA) according to the supplier's instructions. RNA was stored at $-70^{\circ} \mathrm{C}$ until analysis. For quality control, the purity and integrity of the isolated RNA were evaluated using denaturing gel electrophoresis, a spectrophotometer to determine the OD 260/280 ratio, and an Agilent 2100 Bioanalyzer (Agilent Technologies, USA).

DNA microarray hybridization and statistical analysis of microarrayresults. Illumina microarray analysis was performed according to the instructions that were provided for the Illumina Bead station 500X (Illumina, USA). Biotinylated cRNA was generated using the Illumina amplification kit (Ambion, USA) according to the manufacturer's protocol. Briefly, $550 \mathrm{ng}$ of total-RNA was reverse-transcribed to synthesized cDNA using a T7 oligo(dt) primer. Second-strand cDNA was synthesized, in vitro transcribed, and labeled with biotin-NTP. The resulting cRNA was purified using the RNeasy kit (Qiagen, USA) according to manufacturer's protocol, and hybridized to the Sentrix Mouse Ref-8 Expression BeadChip (Illumina) for $16-18 \mathrm{~h}$ at $58^{\circ} \mathrm{C}$. Amersham fluorolink streptavidin-Cy3 (GE Healthcare Bio-Sciences, UK) was used for signal. The Illumina BeadArray Reader confocal scanner was also used. The scanned images were analyzed using Illumina BeadStudio (v. 2.1.12). The microarray experiments on independent ES samples were performed in duplicate.

For data analysis, a filtering criterion was applied to minimize the effect of random and systematic variations. Data were filtered using a detection P-value $<0.05$ in $\geq 50 \%$ samples. Selected gene signal values were transformed and normalized using the logarithmic and quantile methods, respectively. Hierarchical clustering was performed using the Multi Experiment Viewer (18) (http://www.tm4.org/). The comparative analysis was performed using the LPE-test (19) and the fold changes were determined (fold $>2$ or $<2$ ). To prevent potential increases in the error rate of multiple tests, the P-value was adjusted using the Benjamini-Hochberg false discovery rate (FDR) (20). The data discussed in this publication have been deposited in NCBI gene expression omnibus (GEO) and are accessible through GEO series accession number GSE31031 (http://www.ncbi.nlm.nih. gov/geo/query/acc.cgi?acc=GSE31031).

RT-PCR and quantitative reverse transcription-polymerase chain reaction ( $q R T-P C R)$. Reverse transcription was performed with Maxime RT PreMix kit (iNtRON, Korea) to verify ES cell differentiation. For cDNA synthesis, $1 \mu \mathrm{g}$ of total-RNA was added to a premix containing oligo(dt) primer in a final volume of $20 \mu \mathrm{l}$. The prepared mixture was further incubated at $45^{\circ} \mathrm{C}$ for $1 \mathrm{~h}$, and at $95^{\circ} \mathrm{C}$ for $5 \mathrm{~min}$. The following primers were used neurofilament (NF) (forward) 5'-GCAGACATTGCCTCCT ACC-3' and (reverse) 5'-TCACTCCTTCCG TCACCC-3'; bone morphologic protein 4 (BMP4) (forward) 5'-ACTGCCGTCGCC ATTCAC-3' and (reverse) 5'-CACCACCTTGTCATACTCAT 
CC-3'; $\alpha$-fetoprotein (AFP) (forward) 5'-ATTCCTCCCAGTG CGTGAC-3' and (reverse) 5'-GCAGTCTTCTTGCGTGCC-3'; and $\beta$-actin (forward) 5'-TGGAATCCTGTGGCATCCATGAA AC-3' and (reverse) 5'-TAAAACGCAGCTCAGTAACAGT CCG-3'.

For qRT-PCR, the samples were analyzed using the Applied Biosystems 7900HT Fast Real-Time PCR system (Applied Biosystems, UK) with the QuantiTect SYBR-Green PCR kit (Qiagen). Data were analyzed using the $2^{-\Delta \Delta C t}$ method to obtain the relative expression level, which was normalized to the glyceraldehyde-3-phosphate dehydrogenase (GAPDH) expression in each sample.

Restriction-PCR methylation analysis. Genomic DNA was prepared from ESN, ESP and ESH cells using the Gentra Puregene DNA purification kit (Qiagen). All DNA samples were cleaved with $B a m \mathrm{HI}$ to reduce their size and facilitate HhaI, HpaII or MspI digestion and PCR. A total of $600 \mathrm{ng}$ of this DNA was split between two reaction tubes, one of which was incubated with an appropriate methyl-sensitive enzyme at $37^{\circ} \mathrm{C}$ for $12 \mathrm{~h}$. The DNA was then analyzed by quantitative real-time PCR using the Applied Biosystems 7900HT Fast Real-Time PCR System (Applied Biosystems) with the QuantiTect SYBR-Green PCR kit (Qiagen). The following primers were used Hh2 (forward) 5'-CCATAGTCATGGGCT TCA-3' and (reverse) 5'-GGTCAGGTGTCCTCATAG-3'; Hh3 (forward) 5'-CATCTATGAGGACACCTGAC-3' and (reverse) 5'-GGCTTGATGTAGGATTCCTC-3'; Hh4 (forward) 5'-CTG GCTGGTTTGTGGCAGATA-3' and (reverse) 5'-ACTTTGGC TGTCTCTGGAAC-3';Hh5 (forward) 5'-GGCATAGAAGCTG TTATGTGC-3' and (reverse) 5'-GGTTCAGTGTGTAAGGGA ACC-3'; Hh7 (forward) 5'-CTTGGGTAGCTCCTTCAGTC-3' and (reverse) 5'-CTCCTAGTCTCTAATCTCAGC-3'; Hp7 (forward) 5'-CTTTCAACAGAAGCTAAGAGCAAT-3' and (reverse) 5'-GGGCACTCCTCACCGTCCCT-3'; and Hp8 (forward) 5'-AGCAGGCTACGGGGCTATATG-3' and (reverse) 5'-CTCTGTCAACCAATCAGTACATG-3'. Each PCR cycle consisted of $1 \mathrm{~min}$ at $94^{\circ} \mathrm{C}, 1 \mathrm{~min}$ at $56^{\circ} \mathrm{C}$ (for sites $\mathrm{Hh} 2$ and $\mathrm{Hh} 3$ ) or $60^{\circ} \mathrm{C}$, and $1 \mathrm{~min}$ at $72^{\circ} \mathrm{C}$. Data were analyzed using the $2^{-\Delta \Delta \mathrm{C}}$ method to determine the digestion level of DNA that was treated with the methylation-sensitive enzyme relative to that of the uncut DNA.

Generation of chimeric mice using the ESP and ESH cells and growth assays of mouse embryonic fibroblast (MEF) cells derived from chimeric fetuses. To generate chimeric mice using the ESP and ESH cells, approximately 15-20 ES cells were injected into the blastocoels of host blastocysts using a Piezo microinjector (Nikon Instruments Inc., USA). Injected blastocysts were transferred into the uterine horns of 2.5 day postcoitum (d.p.c) recipient mice at 1-4 $\mathrm{h}$ after ES cell injection. Chimeras were identified by coat color.

Primary MEF cells were established from 13.5 d.p.c. chimera fetuses and immediately cultured in a selection media containing $1,000 \mathrm{ng} / \mathrm{ml}$ puromycin to isolate the ES cell with integrated lentiviral vectors. The purity of the H19 knockdown MEF cultures was evaluated based on relative GFP expression and the imprinted gene expression. For the MEF growth assay, puromycin-selected MEFs were plated at concentrations of $10^{4}$ cells per well and counted every other day in triplicate.
Statistical analysis. All graphed data were calculated as the means \pm SD, unless otherwise stated, and from a minimum of three separate experiments. Results were analyzed using an unpaired t-test.

\section{Results and Discussion}

Alteration of the expression of muscle generating and imprinting genes through Igf 2 overexpression and H19 downregulation in parthenogenetic stem cells. ESN, ESP, ESI and ESH cells were established and used to identify differences in the expression of muscle generating and imprinting genes (Fig. 1A). Two ESI and three ESH cell lines were selected from among the ESP cell lines using selection media with puromycin, and these cells were propagated for $>50$ passages. The established ESP cell lines had normal diploid chromosomes and showed typical ES cell surface marker expression and a high level of alkaline phosphatase activity (data not shown).

We performed microarray analysis to evaluate the gene expression patterns of imprinting and muscle-generating genes in each type of ES cell line. Hierarchical clustering analysis was applied to 57 imprinting and 9 muscle-generating genes such as myogenic differentiation antigen 1 (MyoD1), myogenic factor 4 (MYOG), myogenic factor 5 (MYF5), myogenic factor 6 (MYF6) and paired box gene 3 (PAX3). The expression patterns of ESI and ESH cells were similar to and more closely grouped with those of ESN than to the ESP cell (Fig. 1B). In addition, after 10 days of EB formation, the differentiated state of the ES cell lines was confirmed using germ layer specific markers including NF for ectoderm, BMP4 for mesoderm and AFP for endoderm. The ESH cells displayed increased expression of BMP4 compared with the ESI and ESP cells (Fig. 1C). The Igf2 and H19 genes may participate in embryonic development that is associated with the mesoderm $(13,15)$. However, Igf2 expression induces tumorigenesis, and transgenic mice that have been produced from ES cells with the transgene for Igf2 overexpression display symptoms of Beckwith-Wiedemann syndrome (BWS) $(21,22)$. Moreover, the $\mathrm{H} 19$ gene is a non-coding RNA of unknown function that is highly expressed in mesoderm- and endoderm-derived tissues during embryonic development (23) and is a transregulator of the imprinted gene network (IGN), which in involved in embryonic development in mice (24). Recently, Ragian et al (25) suggested that teratomas derived from downregulation of H19 in ESP cells increased mesoderm-derived muscle and endoderm similar to that derived from ESN cells. Therefore, we suggest that the ESP cells have the potential to be used in cell-based therapies to treat muscular atrophy and that the ESH cells may be more effective than the ESI cells.

H19 silencing revealed linked expression of H19 and Igf2imprinted genes and H19 imprinting control region (ICRI) DNA methylation. To confirm the relationship between the H19 and Igf2 expression levels and the dose-dependent influence of H19 expression on Igf2 expression, the ESN, ESP and ESH cells with different levels of $\mathrm{H} 19$ expression were differentiated using an EB formation protocol for 10 days. The differentiated ES cells were simultaneously analyzed by qRT-PCR (Fig. 2A). All of the ESH cell lines showed an increased Igf2 expression and decreased H19 expression compared with the ESP cells. Mice that have 

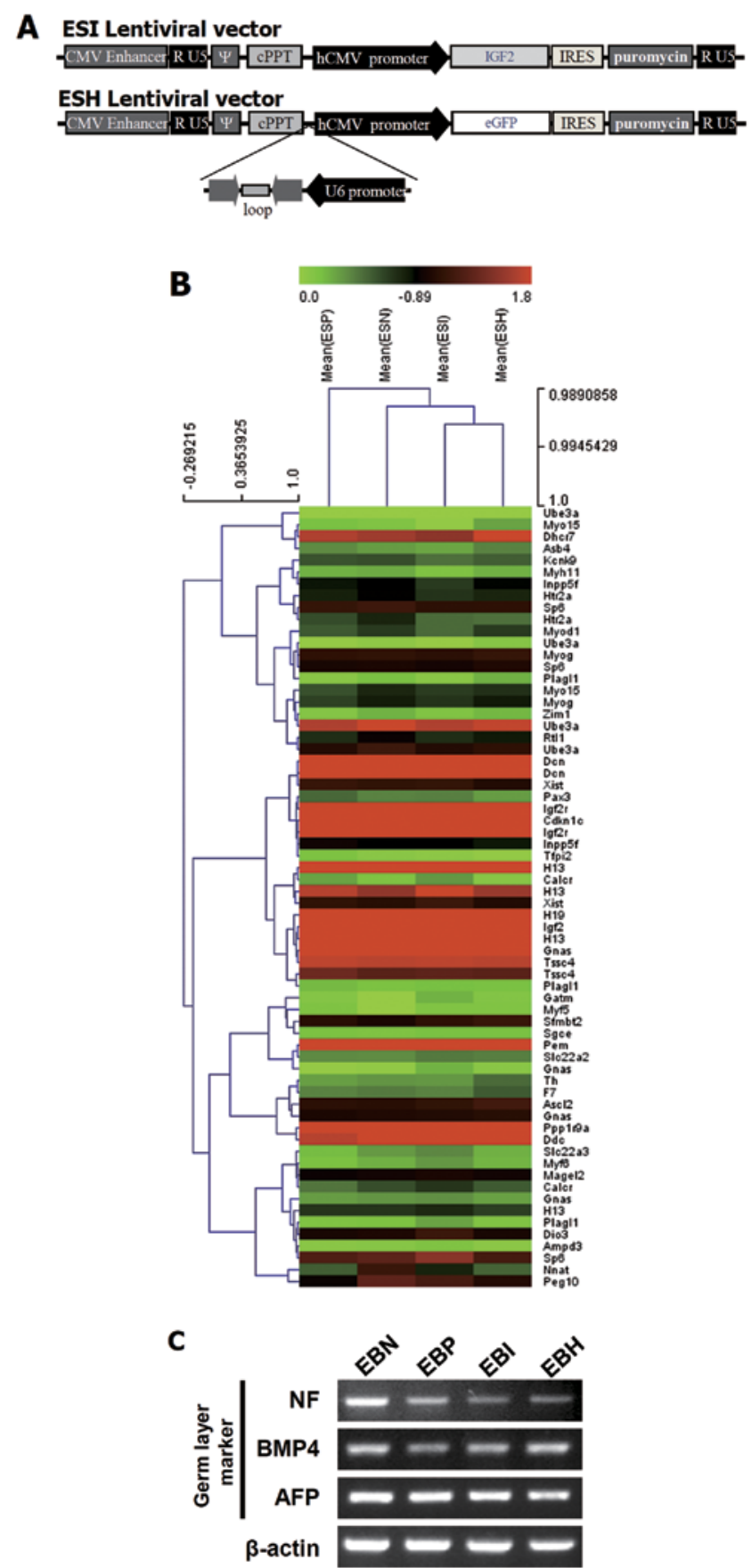

Figure 1. Development of ESI and ESH cell lines. (A) Lentiviral vector construction to establish ESI and ESH cell lines. Igf2 and puromycin expression in the ESI lentiviral vector was controlled by the hCMV promoter. The ESH lentiviral vector was a self-inactivating lentiviral vector containing the cPPT gene and a central termination sequence followed by the U6 promoter, which mediated shH19 expression, and the hCMV promoter, which mediated eGFP and puromycin expression. (B) The expression profiles of 57 imprinting genes and 9 genes that were associated with muscle generation in the established ESN, ESP, ESI and ESH cell lines. (C) RT-PCR results of germline-specific genes [neurofilament (NF), bone morphologic protein 4 (BMP4) and $\alpha$-fetoprotein (AFP)].

a deletion in the H19 transcribed region show an overgrowth phenotype that is concomitant with increased Igf 2 expression in mesoderm-derived tissues including muscle, tongue, diaphragm and heart; this deletion also significantly affected six imprinting genes including IGN $(24,26)$. Among these genes, the H19 RNA has inhibitory effects on Igf 2 transcription. In this study, we observed a similar phenomenon, even with siRNA-mediated $\mathrm{H} 19$ silencing. In addition, the expression of the Igf2/H19 locus is controlled by the methylation status of differentially-methylated region 2 (DMR2), ICR1 and the H19 promoter (27). Methylated ICR1 binds to the CCCTC binding factor (CTCF), which is the major insulator protein in vertebrates, and CTCF that has bound to ICR1 prevents Igf2 transcription (28). Therefore, to understand the effect of $\mathrm{H} 19$ silencing on the regulation of H19-ICR1 methylation, we analyzed the methylation state of the $2-\mathrm{kb}$ H19-ICR1 region, which is located in the 5' flank of the mouse H19 gene. The methylation of the H19-ICR1 region is increased in the paternal chromosome compared to that in the maternal chromosome in post-implantation embryos (29) and modulates the enhancer activity of $\mathrm{H} 19$, which regulates Igf 2 expression.

To determine whether the methylation state of H19-ICR1 was altered, methylation changes at $7 \mathrm{CpG}$ sites within the H19-ICR1 region [Hh2, Hh3, Hh4, Hh5, Hh7, Hp7 and Hp8 (30)] were studied using a methylation-sensitive restriction enzyme-PCR assay (Fig. 2B and C). The ESN, ESP and ESH cells were differentiated for 10 days using the EB formation protocol, because the undifferentiated ES cells exhibit only a basal level of methylation throughout the genomic DNA. Using quantitative PCR analysis of each differential methylation site (Hh2-Hp8) of differentiated cells, we demonstrated that the methylation level was lower at each site in the ESP cells than in the ESN cells. However, the ESH cells had a significantly elevated level of methylation compared with the ESP cells in all of the analyzed sites. At many of the sites (Hh2, Hh3, Hh7, $\mathrm{Hp} 7$ and $\mathrm{Hp} 8$ ), the methylation levels of the ESH cells were higher than those of the ESN cells. After cutting the $\mathrm{Hp} 7$ or Hp8 sites with MspI (an isoschizomer of HpaII) as a control, no differences were detected between the three cell lines, indicating that the sites were efficiently cut (this result is difficult to see in the graph because of the extremely low values). These results indicate that $\mathrm{H} 19$ silencing influences the methylation recovery of the H19-ICR1 region, which prevents CTCF from binding to the ICR1. In this case, the enhancers downstream of the H19 locus may activate the Igf2 gene, a mechanism similar to that of the paternal allele in the ESN cells.

The exact mechanism by which the H19 RNA regulates the methylation state of the H19-ICR1 region is not yet understood. However, it was recently discovered the H19 RNA functions as a primary microRNA transcript (31). The H19 RNA may regulate DNA methylation mechanisms as a microRNA. Therefore, it would be valuable to determined whether the H19-derived microRNA target transcripts are involved in DNA methylation.

Generation of chimeric mice and enhanced growth rates of the MEF cells resulting from $H 19$ silencing. To functionally validate the up-regulation of Igf2 in the ESH cells, chimeric mice were produced by injecting the ESP and ESH cells and were identified chimerism rate by coat color (Table I). The proportion of chimeras that were produced from the ESH cells was slightly higher than that of chimeras that were produced from the ESP cells. The MEF cells were isolated from GFP-expressing chimeric fetuses because the contribution of ESH and ESP cells to skeletal muscle was approximately 5\% and was similar in each group (data not shown). To separate the chimeric fetus-derived MEFs, isolated MEF cells were 
A

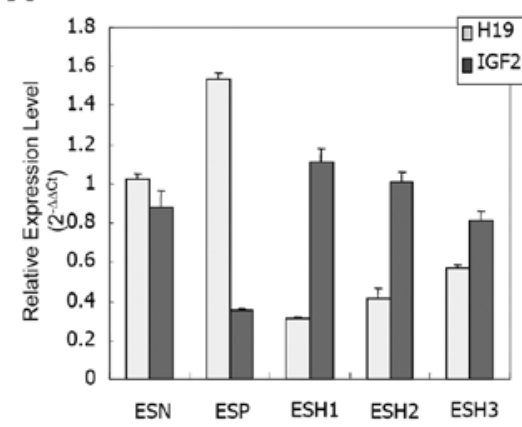

C

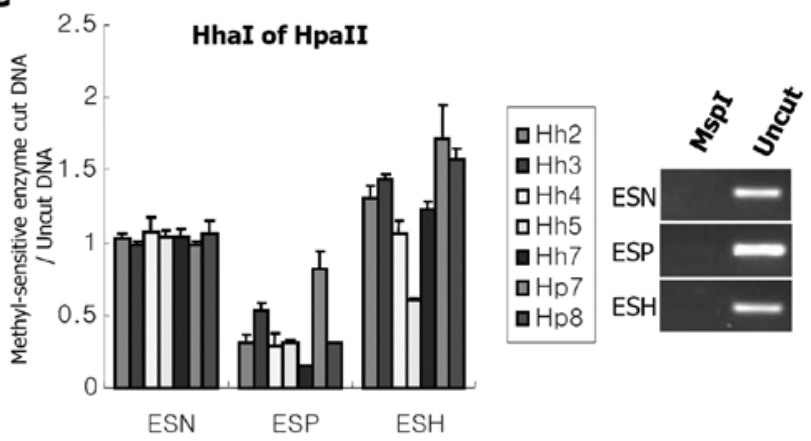

B

H19-ICR1

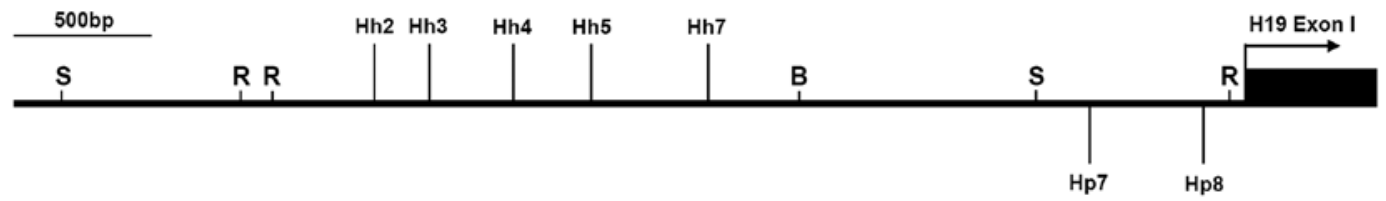

Figure 2. Linked expression of the H19 and Igf2 imprinting genes and H19-ICR1 methylation recovery by H19 silencing. (A) Quantitative RT-PCR analysis revealed increased H19 expression and decreased Igf2 expression in the ESP cell derivatives (EB cells after 10 days of differentiation). All of the ESH cell derivatives exhibited Igf2 up-regulation and H19 down-regulation. (B) A map of the region and positions of methylation-sensitive enzyme sites. The HhaI (Hh) and HpaII (Hp) sites are indicated above and below the line, respectively, and are numbered according to the convention of Ueda et al (33). (C) The restriction-PCR methylation assays for the HhaI and HpaII sites were performed using a real-time PCR method after 10 days of EB differentiation using ESN, ESP and ESH cells. All of the analyzed sites were re-methylated after H19 silencing (left panel, n=3, P<0.01). Hp7 sites were analyzed after the MspI digestion as control for an enzymatic digestion (right panel).

Table I. Production of chimeric mice using ESP and ESH cells.

\begin{tabular}{lccc}
\hline $\begin{array}{l}\text { Type of parthenogenetic } \\
\text { stem cell }\end{array}$ & $\begin{array}{c}\text { Live births } \\
\mathrm{n}\end{array}$ & $\begin{array}{c}\text { Chimeric pups } \\
\mathrm{n}(\%)\end{array}$ & $\begin{array}{c}\text { Female chimeras } \\
\mathrm{n}\end{array}$ \\
\hline ESP $^{\mathrm{a}}$ & 72 & $18(25)$ & 8 \\
ESH $^{\mathrm{b}}$ & 46 & $15(32.6)$ & 7 \\
\hline
\end{tabular}

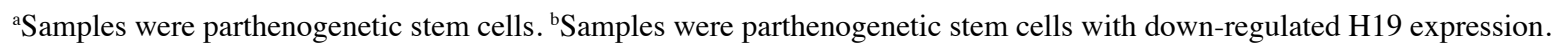
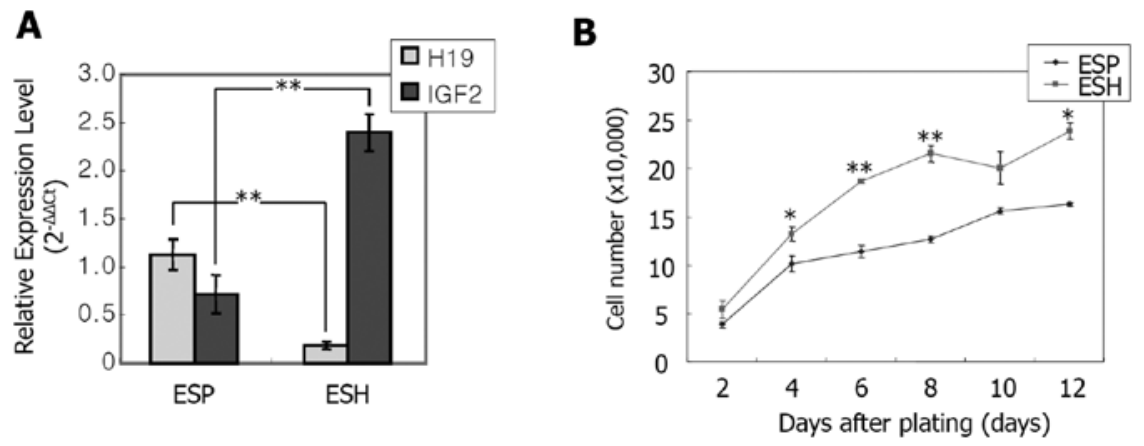

Figure 3. Enhanced growth of H19 down-regulated MEF cells. (A) The ESH cell-derived MEFs showed significant down-regulation of H19 and up-regulation of Igf2 compared with GFP-ESP cell-derived control MEFs. (B) The ESH cell-derived MEF cells grew faster than GFP-ESP cell-derived MEF cells ( $\mathrm{n}=4$ ). $\left({ }^{*} \mathrm{P}<0.05 ;{ }^{* *} \mathrm{P}<0.01\right)$.

cultured using selection media containing puromycin. The purity of the ES-derived MEFs was determined using two methods. First, the GFP expression levels of the ESP and ESH cell lines were measured by RT-PCR analysis because the puromycin-selected MEF cells showed intense GFP expression (data not shown). Second, the H19 and Igf2 gene expression levels of the selected MEF cells were analyzed by qRT-PCR to confirm whether the selected MEF cells were representative of the typical gene expression pattern (Fig. 3A). The ESH-derived MEF cells showed H19 down-regulation 
and Igf2 up-regulation due to H19 silencing effects. Therefore, we assumed that the differences in the actual expression levels of H19 and Igf2 were due to the differences in the cell types. Igf2 is an important mitogen during normal fetal growth (32). Therefore, to identify changes in cell proliferation that were induced by increased Igf2 expression via the H19 silencing, the proliferation of the ESP-MEF and ESH-MEF cell lines was measured using cells at the third passage. The two lines of ESH-MEF cells consistently proliferated at a faster rate than the two lines of ESP-MEF cells (Fig. 3B). Increased Igf 2 expression enhanced the growth rate of the cells, which confirmed the role of Igf 2 as a functional and effective mitogen. Consequently, our results suggest that the muscle of patients with muscular atrophy may be restored using parthenogenetic stem cells that overexpress Igf2 due to H19 silencing.

\section{Acknowledgements}

This study was supported by the Basic Science Research Program through the National Research Foundation of Korea (NRF), funded by the Ministry of Education, Science and Technology (2009-0063816).

\section{References}

1. Evans MJ and Kaufman MH: Establishment in culture of pluripotential cells from mouse embryos. Nature 292: 154-156, 1981.

2. Gutierrez-Aranda I, Ramos-Mejia V, Bueno C, et al: Human induced pluripotent stem cells develop teratoma more efficiently and faster than human embryonic stem cells regardless the site of injection. Stem Cells 28: 1568-1570, 2010.

3. Hao J, Zhu W, Sheng C, Yu Y and Zhou Q: Human parthenogenetic embryonic stem cells: one potential resource for cell therapy. Sci China C Life Sci 52: 599-602, 2009.

4. Revazova ES, Turovets NA, Kochetkova OD, et al: HLA homozygous stem cell lines derived from human parthenogenetic blastocysts. Cloning Stem Cells 10: 11-24, 2008.

5. Brevini AL and Gandolfi F: Parthenotes as a source of embryonic stem cells. Cell Prolif 41: 20-30, 2008.

6. Emery AE: The muscular dystrophies. Lancet 359: 687-695, 2002.

7. Meregalli M, Farini A, Parolini D, Maciotta S and Torrente Y: Stem cell therapies to treat muscular dystrophy: progress to date. BioDrugs 24: 237-247, 2010.

8. Bhagavati $\mathrm{S}$ and $\mathrm{Xu} \mathrm{W}$ : Generation of skeletal muscle from transplanted embryonic stem cells in dystrophic mice. Biochem Biophys Res Commun 333: 644-649, 2005.

9. Kamochi H, Kurokawa MS, Yoshikawa H, et al: Transplantation of myocyte precursors derived from embryonic stem cells transfected with IGFII gene in a mouse model of muscle injury. Transplantation 82: 516-526, 2006.

10. Allen ND, Barton SC, Hilton K, Norris ML and Surani MA: A functional analysis of imprinting in parthernogenetic embryonic stem cells. Development 120: 1473-1482, 1994.

11. Horii T, Kimura M, Morita S, Nagao Y and Hatada I: Loss of genomic imprinting in mouse parthenogenetic embryonic stem cells. Stem Cells 26: 79-88, 2008.

12. Kono T, Obata Y, Wu Q, et al: Birth of parthenogenetic mice that can develop to adulthood. Nature 428: 860-864, 2004.
13. Morali OG, Jouneau A, McLaughlin KJ, Thiery JP and Larue L: IGF-II promotes mesoderm formation. Dev Biol 277: 133-145, 2000.

14. Lopes S, Lewis A, Hajkova P, et al: Epigenetic modifications in an imprinting cluster are controlled by a hierarchy of DMRs suggesting long-range chromatin interactions. Hum Mol Genet 12: 295-305, 2003.

15. Merrick D, Ting T, Stadler LK and Smith J: A role for insulinlike growth factor 2 in specification of the fast skeletal muscle fiber. BMC Dev Biol 7: 65, 2007.

16. Murphy SK, Huang Z, Wen Y, et al: Frequent IGF2/H19 domain epigenetic alterations and elevated IGF2 expression in epithelial ovarian cancer. Mol Cancer Res 4: 283-292, 2006.

17. Bryja V, Bonilla S, Cajánek L, et al: An efficient method for the derivation of mouse embryonic stem cells. Stem Cells 24: 844-849, 2006

18. Saeed AI, Sharov V, White J, et al: TM4: a free, open-source system for microarray data management and analysis. Biotechniques 34 : 374-378, 2003.

19. Jain N, Thatte J,Braciale T,Ley K, O'Connell M and Lee JK: Localpooled-error test for identifying differentially expressed genes with a small number of replicated microarrays. Bioinformatics 19: 1945-1951, 2003.

20. Benjamin Y and Hochberg Y: Controlling the false discovery rate, a practical and powerful approach to multiple testing. J Roy Statist Soc 57: 289-300, 1995.

21. Christofori G, Naik P and Hanahan D: A second signal supplied by insulin-like growth factor II in oncogene-induced tumorigenesis. Nature 369: 414-418, 1994.

22. Sun FL, Dean WL, Kelsey G, Allen ND and Reik W: Transactivation of Igf 2 in a mouse model of Beckwith-Wiedemann syndrome. Nature 389: 809-815, 1997.

23. Gabory A, Jammes H and Dandolo L: The H19 locus: role of an imprinted non-coding RNA in growth and development. Bioessays 32: 473-480, 2010.

24. Gabory A, Ripoche MA, Le Digarcher A, et al: H19 acts as a trans regulator of the imprinted gene network controlling growth in mice. Development 136: 3413-3421, 2009.

25. Ragina NP, Schlosser K, Knott JG, et al: Down-regulation of H19 improves the differentiation potential of mouse parthenogenetic embryonic stem cells. Stem cells Dev: Sep 14 (Epub ahead of print), doi:10.1089/scd.2011.0152, 2011.

26. Yoshimizu T, Miroglio A, Ripoche MA, et al: The H19 locus acts in vivo as a tumor suppressor. Proc Natl Acad Sci USA 105: 12417-12422, 2008.

27. Tabano S, Colapietro P, Cetin I, et al: Epigenetic modulation of the IGF2/H19 imprinted domain in human embryonic and extraembryonic compartments and its possible role in fetal growth restriction. Epigenetics 5: 313-324, 2010.

28. Lee DH, Singh P, Tsark WM and Szabó PE: Complete biallelic insulation at the H19/Igf2 imprinting control region position results in fetal growth retardation and perinatal lethality. PLoS One 5: e12630, 2010.

29. Bartolomei MS, Webber AL, Brunkow ME and Tilghman SM: Epigenetic mechanisms underlying the imprinting of the mouse H19 gene. Genes Dev 7: 1663-1673, 1993.

30. Tremblay KD, Saam JR, Ingram RS, Tilghman SM and Bartolomei MS: A paternal-specific methylation imprint marks the alleles of the mouse H19 gene. Nat Genet 9: 407-413, 1995.

31. Smits G, Mungall AJ, Griffiths-Jones S, et al: Conservation of the H19 noncoding RNA and H19-IGF2 imprinting mechanism in therians. Nat Genet 40: 971-976, 2008.

32. Constância M, Hemberger M, Hughes J, et al: Placental-specific IGF-II is a major modulator of placental and fetal growth. Nature 417: 945-948, 2002.

33. Ueda T, Abe K, Yuzuriha M, et al: The paternal methylation imprint of the mouse $\mathrm{H} 19$ locus is acquired in the gonocyte stage during foetal testis development. Genes Cells 5: 649-659, 2000. 